Please do not remove this page

RMIT

UNIVERSITY

\title{
Influence of sinusoidally modulated visual stimuli at extremely low frequency range on the human EEG Activity
}

Cvetkovic, Dean; Simpson, David; Cosic, Irena

https://researchrepository.rmit.edu.au/esploro/outputs/9921862620401341/filesAndLinks?institution=61RMIT_INST\&index=null

Cvetkovic, D., Simpson, D., \& Cosic, I. (2006). Influence of sinusoidally modulated visual stimuli at extremely low frequency range on the human EEG Activity. 28th IEEE Engineering in Medicine and Biology Society (EMBS) Annual International Conference. https://doi.org/10.1109/IEMBS.2006.259565

Published Version: https://doi.org/10.1109/IEMBS.2006.259565

Repository homepage: https://researchrepository.rmit.edu.au

(c) 2006 IEEE. Personal use of this material is permitted. However, permission to reprint/republish this material for advertising or promotional purposes or for creating new collective works for resale or redistribution to servers or lists, or to reuse any copyrighted component of this work in other works must be obtained from the IEEE.

Downloaded On 2023/04/27 00:57:53 +1000 


\title{
Influence of Sinusoidally Modulated Visual Stimuli at Extremely Low Frequency Range on the Human EEG Activity
}

\author{
D. Cvetkovic, D. Simpson and I. Cosic, Senior Member
}

\begin{abstract}
The purpose of this study was to investigate whether sinusoidally modulated visual stimuli at extremely low frequencies (ELF) of 50, 16.66, 13, 10, 8.33 and $4 \mathrm{~Hz}$ could influence the changes in EEG activity in 33 human subjects. An improved design of visual stimulator system has addressed an issue of electrical interference from electrical signals driven by LED arrays onto simultaneously recorded EEG. A comparison between 1 and 3-Way ANOVA was performed in order to evaluate whether visual stimuli at ELFs could influence the EEG in humans to compliment the currently active medical research in seasonal affective disorder (SAD) and photic driving. The results revealed that under evaluation of 1 and 3-Way repeated-measures ANOVA tests, the Theta, Alpha2 and Gamma EEG bands exhibited a common significant difference between ELF visual stimuli.
\end{abstract}

\section{INTRODUCTION}

$\mathrm{O}$ ver the last several decades, research results from various studies have shown that visible light, represented by a narrow band of the electromagnetic spectrum and integrated with geomagnetic field, can influence the circadian rhythms and biological sleep-wake cycles in humans, animals [1]-[3] and plants [4]. The pineal gland of animals and humans is a neural structure that is functionally related to the visual system. The light-dark alteration in the retina could produce responsive suppression of pineal melatonin synthesis (light exposure period) while the maximum production and secretion of the hormone occurs during the dark periods (night time exposure period).

Deprivation or lack of sunlight has shown to cause seasonal affective disorder (SAD) in humans during the winter season. SAD may either alter a circadian phase relationships or sleep-wake dependant processes. As a result of these alterations, a change of daytime energy and

Manuscript received April 3, 2006.

D. Cvetkovic (corresponding author) is with RMIT University, School of Electrical and Computer Engineering, GPO Box 2476V, Melbourne, VIC 3001, Australia, tel: +613 9925 9641, fax: +613 9925 2007, e-mail: dean.cvetkovic@rmit.edu.au.

D. Simpson is with Brain Sciences Institute, Swinburne University, PO Box 218 Hawthorn, VIC 3122, Australia, tel: +6139214 5212, fax: +613 9214 5525, e-mail: dsimpson@swin.edu.au.

I. Cosic is with RMIT University, School of Electrical and Computer Engineering, GPO Box 2476V, Melbourne, VIC 3001, Australia, tel: +613 9925 1971, fax: +613 9925 2007, e-mail: irena.cosic@ rmit.edu.au. sleepiness levels may occur [5]. Much is known concerning the ability of light to affect the stability of a person's energy, well-being, sleep, moods, body temperature, concentration and the regulation of a person's circadian rhythms [2]. The use of light therapy may treat physical and emotional problems, such as patients that have been afflicted with a seasonal affective disorder. When mammals and humans are exposed in the photo-periodic environment, significant alterations to the circadian system, production of neuro-hormone melatonin and EEG activities may be evident [6].

The 'photic' driving response is usually referred to as the 'steady-state evoked potential' [7]. The term 'driving response' has been defined as an occurrence of waves of the same frequency or of waves that are harmonically related to the frequency of the rhythmic stimuli (phase-locked). This technique states that the brain is being presumed to have achieved a steady state of excitability. It has been suggested that the photic driving response is a resonance between spontaneous rhythms of the brain and visually evoked responses.

One study clearly indicated the possibility of 'entraining' (alternative term for photic driving) specific EEG frequency bands by presenting subjects with frequency-specific flickering lights [7]. In response to repetitive flashes, the induced rhythms could be confirmed as long as the stimuli frequency is below $10 \mathrm{~Hz}$. The EEG power spectrum to visual stimulation showed entrainments around the Alpha frequency. However, it was also reported that one-half of their subjects did not produce a photic driving response within the Alpha band-pass to fixed frequency, repetitive stroboscopic flashes ranging from 5 to $16 \mathrm{~Hz}$.

Another study was conducted to examine sex differences in inter-hemispheric EEG coherence in 15 male and 15 female healthy young adults during rest and visual stimulation at 5,10 and $15 \mathrm{~Hz}$ [8]. The results revealed a significant gender related differences found in EEG coherence during visual stimulation, where females had a significantly higher coherence than the males at frontal and central regions in the frequency band $(4.5-5.5 \mathrm{~Hz})$ corresponding to $5 \mathrm{~Hz}$ visual stimulation. Also, females had significantly greater coherence reactivity at occipital region in EEG during 5 and $15 \mathrm{~Hz}$. Overall, the results indicated a gender related differences in inter-hemispheric EEG coherence during visual stimulation.

The purpose of this study was to investigate whether sinusoidally modulated stimuli at extremely low frequencies 
of $50,16.66,13,10,8.33$ and $4 \mathrm{~Hz}$ could influence the changes in EEG activity in 33 human subjects by performing 1 and 3-Way ANOVA statistical tests $[9,10]$. Any possible significant differences between the stimuli responses on the EEG bands could contribute towards better understanding of photic driving theory.

\section{MATERIALS AND METHODS}

\section{A. Design of Visual Stimulator}

The Visual Stimulator consisted of a light generated by arrays of light emitting diodes (LEDs). There were 2 LEDs placed in individual miniature double-Faraday cages for electrical-field shielding. The cages were attached to a goggle so that each LED arrays is placed directly over each eye becoming the sole stimuli. The LED arrays emit red light allowing easy viewing through closed eyelids. The main causes of electrical interference were the electrical signals driving the LED arrays, which could easily be detected in the EEG recordings during subject stimulation. Therefore, double Faraday cages were implemented to house the LED arrays shielding the recorded brain waves from this electrical interference. In initial testing with no shielding surrounding the LED arrays, the modulation signal was easily detectable in the recorded EEG. Reduced electrical interference was still detectable while using a single shield surrounding the LED arrays. However, when the LED arrays were shielded by two separate Faraday cages (a double Faraday cage), synchronous averaging measurements were unable to detect any interference from the LED arrays or inter-connecting leads. The two shields of the Faraday-cages were shorted at the start of the cable supplying the drive-voltage to the LED arrays.

The sinusoidally modulated stimuli and a sinusoidally modulated luminance flicker using LED arrays is utilised to conduct Steady State Visual Stimulations (SSVS). The Steady State Evoked Potentials (SSVEP) are elicited by a repetitive sinusoidal stimuli $[11,12]$. To overcome the inherent non-linearity of LED luminance (brightness per unit area) and LED driving signal an electronic luminance-linearising circuit was developed. In a darkened room, the luminance of the LED array was measured using a TEKTRONIX J16 Digital Photometer with a TEKTRONIX J6523-2 1 degree Narrow Angle Luminance Probe. Measurements showed a better than $0.5 \%$ linearity error between the input driving signal and output luminance of the LED array.

To conduct these measurements, the LED luminance lineariser circuit was driven by various static voltage signals while luminance measurements were tabulated. E Tabor Electronics (20MHz) Programmable Function Generator (Series 8200) was pre-programmed with $50 \mathrm{~Hz}$, $16.66 \mathrm{~Hz}, 13 \mathrm{~Hz}, 10 \mathrm{~Hz}$ and $4 \mathrm{~Hz}$ frequencies, DC offset of $0.75 \mathrm{~V}$ and amplitude of $1.5 \mathrm{~V}$ to generate sinusoidally modulated photic stimuli using LEDs and presented to subject's eyes. The light emittance (Lux) of one array of LEDs from one Faraday cage, positioned approximately
$2.5 \mathrm{~cm}$ from subject's eyes was measured with light meter (D. Smith) and the light emittance was measured to be 50 Lux.

\section{B. Subjects and EEG Montages and Procedures}

The experiments were conducted on 33 healthy subjects, 24 male and 9 female, with mean age of 30 years, SD 11 years, range $20-59$ years. The RMIT ethics committee approved the study and all subjects gave written informed consent prior to the experiment. During the EEG recording sessions, subjects were asked to lie down in the supine position. The entire experiment was performed in a darkened and sound proof RF anechoic chamber to prevent erroneous recordings due to the standing waves and power line interference.

\section{C.EEG Recording and Experimental Protocol}

The EEG equipment used throughout testing was the Mindset MS-1000 recording system. Neuroscan 19 Channel Caps electrodes were used with referential montage of 16 channels. The left brain hemisphere electrodes: Fp1, F7, F3, T7, C3, P7, P3 and O1 were all referenced to M1 (left mastoid), while the right brain hemisphere electrodes: Fp2, $\mathrm{F} 8, \mathrm{~F} 4, \mathrm{~T} 8, \mathrm{C} 4, \mathrm{P} 8, \mathrm{P} 4$ and $\mathrm{O} 2$ were referenced to right mastoid M2. The baseline EEG was recorded prior to stimulation for one minute.

The participant's eyes remained closed throughout the stimulation period. The intensity of light emitted from LED spectacles was individually adjusted to each participant's comfort level prior to any stimulation and EEG sessions. During visual stimulation, each participant's brain electrical activity data was recorded. The visual stimulation experiment consisted of recording of the EEG signals prior to any stimulation (baseline EEG 1 minute epoch recording), followed by $1 \mathrm{st}$ VS session (1 minute) at $50 \mathrm{~Hz}$ and simultaneous 2nd EEG recording (1 minute epoch). The EEG recordings were repeated during $16.66 \mathrm{~Hz}, 13 \mathrm{~Hz}, 10 \mathrm{~Hz}$, $8.33 \mathrm{~Hz}$ and $4 \mathrm{~Hz}$ VS sessions. An extra (final post-stimulation) EEG recording undertaken at 1-minute epoch after the last $4 \mathrm{~Hz}$ VS. The total time for VS session was 14 minutes, which included 8 minutes used for EEG recordings and 6 minutes for VS.

\section{Signal Processing AND Statistical Method}

Recorded data was analysed using the Mindset's 'WaveLab' software. Initially, each raw EEG recording was observed for artifacts. Any obvious ocular, muscular or any cardiacular artifacts were removed manually. The FFT Blackman Window was used throughout the signal processing. The Blackman window had better accuracy than the Hanning when tested, very good selectivity and fast filter roll-off. Briefly, the FFT histogram was observed to ensure the validity of the recording as well as to note any generic trends. Numerical FFT data was imported into an Excel spreadsheet for collation and statistical analysis. Extracted FIR filtered data between 0.5 and $42 \mathrm{~Hz}$ at $0.5 \mathrm{~Hz}$ intervals was analysed and the average spectral amplitude was 
performed on each subject's data over 7 frequency ranges or EEG bands: Delta $0.5-3.5 \mathrm{~Hz}$, Theta $4.0-7.5 \mathrm{~Hz}$, Alpha1 $8.0-9.5 \mathrm{~Hz}$, Alpha2 $10.0-12.5 \mathrm{~Hz}$, Beta1 $13.0-16.0 \mathrm{~Hz}$, Beta2 $16.5-31.5 \mathrm{~Hz}$ and Gamma $32.0-42.0 \mathrm{~Hz}$ [11/194]. A summation of the spectral amplitudes within these EEG bands was calculated for VS. These values were then compared and were the main focus upon deciding whether the effects of VS may have caused any changes in the EEG activity of the 33 subjects tested.

\section{Results}

\section{A. Friedman's Rank (1-Way ANOVA) Test}

SPSS statistical tool was utilised to perform One-way repeated-measures analysis of variance, Friedman's Rank Test for $k$ Correlated Samples for statistical analysis of EEG signals. This test is of non-parametric type and is closely related to standard repeated-measures analysis of variance applied to ranks instead of raw score. To apply Friedman Rank test (FR), it was required to rank raw scores for each subject separately and then sum the rankings for each condition. The conditions in this particular instance were stimuli such as baseline, $50 \mathrm{~Hz}, 16.66 \mathrm{~Hz}, 13 \mathrm{~Hz}, 10 \mathrm{~Hz}$, $8.33 \mathrm{~Hz}$ and $4 \mathrm{~Hz}$. Also, each EEG band was evaluated separately using Friedman's test.

The Friedman's test was employed in this case to reject the null hypothesis that averaged spectral amplitudes at each EEG band for each stimulation type did not vary across all conditions (stimulation frequencies) including the baseline (pre-stimulation). In this particular case, the stimulation conditions were tested as within-subjects factor for its significant difference between each other at each EEG band. Friedman's test results revealed that a significant difference was evident for Alpha2 $\left(\mathrm{F} 7, \chi^{2}(6)=24.754, \mathrm{p}<0.000 ; \mathrm{F} 8\right.$, $\chi^{2}(6)=19.073, \mathrm{p}<0.004 ; \mathrm{F} 3, \chi^{2}(6)=30.458, \mathrm{p}<0.0005 ; \mathrm{F} 4$, $\chi^{2}(6)=26.649, \mathrm{p}<0.0005 ; \mathrm{T} 7, \chi^{2}(6)=16.320, \mathrm{p}<0.012 ; \mathrm{T} 8$, $\chi^{2}(6)=20.013, \mathrm{p}<0.003 ; \mathrm{C} 3, \chi^{2}(6)=20.805, \mathrm{p}<0.002 ; \mathrm{C} 4$, $\chi^{2}(6)=33.646, \mathrm{p}<0.0005 ; \mathrm{P} 3, \chi^{2}(6)=20.416, \mathrm{p}<0.002 ; \mathrm{P} 4$, $\chi^{2}(6)=21.390, \mathrm{p}<0.002 ; \mathrm{P} 7, \chi^{2}(6)=19.910, \mathrm{p}<0.003 ; \mathrm{P} 8$, $\chi^{2}(6)=14.753, \mathrm{p}<0.022 ; \mathrm{O} 1, \chi^{2}(6)=30.701, \mathrm{p}<0.0005$; and O2, $\left.\chi^{2}(6)=25.299, \mathrm{p}<0.0005\right)$ and Gamma bands at all electrode positions, as shown in Figure 1. There was no significant difference observed for the Alpha1 and Beta2 bands (except for P8 electrode, $\chi^{2}(6)=23.522, \mathrm{p}<0.001$ ). Delta band exhibited a significant difference at F7, F8, F3, T8, P4 and P7 electrodes. Theta, like Delta bands revealed significant differences at the same electrode positions with additional F4, C4 and P3. Significant differences were observed at electrodes: F7, F8, F3, F4, C4, P3, P4, O1 and $\mathrm{O} 2$ for Beta1 band.

\section{B. 3-Way ANOVA Test}

A three-way ANOVA, where the factors were called A, B and $\mathrm{C}$, were expected to give rise to three main effects (main effect of $A$, main effect of $B$ and main effect of $C$ ), three two-way interaction $\left(A^{*} B, A^{*} C\right.$ and $\left.B^{*} C\right)$ and a single three-way interaction $\left(\mathrm{A}^{*} \mathrm{~B}^{*} \mathrm{C}\right)$. The total of 6 F-test and $\mathrm{p}$-value results were presented for each three-way ANOVA design, where $\mathrm{A}, \mathrm{B}$ and $\mathrm{C}$ factors were represented by A-electrode (16), B-stimuli (7)/bands (7) and C-gender (2). Throughout this investigation, the factor interactions design was $\left(\mathrm{A}^{*} \mathrm{~B}\right) * \mathrm{C}$ and it consisted of two parts. For the first 3-way ANOVA part, the factors tested were described as follows: Within-subject electrode and bands factors with between-subject gender factor $(16 \times 7) \times 2$; and Within-subject electrode and stimuli factors with between-subject gender factor $(16 \times 7) \times 2$. Ten individual computations were undertaken to perform the above ANOVA calculations. These consisted of 5 within-subject band factors and 5 within-subject stimuli factors, describing the N-Way ANOVA methodology and factor combinations. For all the results the degree of freedom was adjusted by Greenhouse and Geisser's epsilon when appropriate.

The 3-way ANOVA (stimuli factor within-subject and gender factor between-subject) revealed a significant difference $(\mathrm{p}<0.05)$ of all the main and interacted factors: electrode $\quad(\mathrm{F}(1.63,50.39)=39.327, \quad \mathrm{p}<0.0005)$; electrode*gender $(\mathrm{F}(1.63,50.39)=4.806, \mathrm{p}<0.018)$; stimuli $(\mathrm{F}(3.79,117.41)=4.933, \quad \mathrm{p}<0.001) ; \quad$ stimuli*gender $(\mathrm{F}(3.79,117.41)=3.382, \mathrm{p}<0.013)$; electrode* stimuli $(\mathrm{F}(90$, $2790)=3.106, \mathrm{p}<0.0005)$; electrode*stimuli*gender $(\mathrm{F}(90$, $2790)=2.28, \quad \mathrm{p}<0.0005) \quad$ and gender $\quad(\mathrm{F}(1,31)=7.739$, $\mathrm{p}<0.009$ ) at Alpha1 band. The other bands, either showed significant differences of electrode and electrode*stimuli or stimuli factors. The band factor within-subject and gender factor between-subject, exhibited a complete significant difference of all factors at all stimulation frequency intervals (stimuli), except at $4 \mathrm{~Hz}$ which revealed non-significant results of electrode*gender $(\mathrm{F}(2.145,66.49)=1.329$, $\mathrm{p}<0.272)$, bands*gender $(\mathrm{F}(2.224,68.96)=1.845, \mathrm{p}<0.162)$ and gender $(\mathrm{F}(1,31)=0.87, \mathrm{p}<0.358)$ factors.

\section{V.DISCUSSION}

The Friedman's Rank Test results revealed that Delta band exhibited a significant difference at all frontal (except F4), right parietal and parietal regions. Theta band revealed significant differences at the same regions, with additional right frontal, right central and left parietal regions. There was no significant difference observed for the Alphal band, except for right parietal (P8) region. A significant difference was evident for the Alpha2 band at all brain regions. Also, significant differences were observed at all frontal, right central, parietal and occipital regions for the Betal band. There was no significant difference observed for the Beta2 band except for right parietal (P8) region. A significant difference was evident for the Gamma band at all brain regions. The 3-way ANOVA model at each EEG band revealed a significant difference at the Theta, Alpha1, Alpha2 and Gamma bands. 


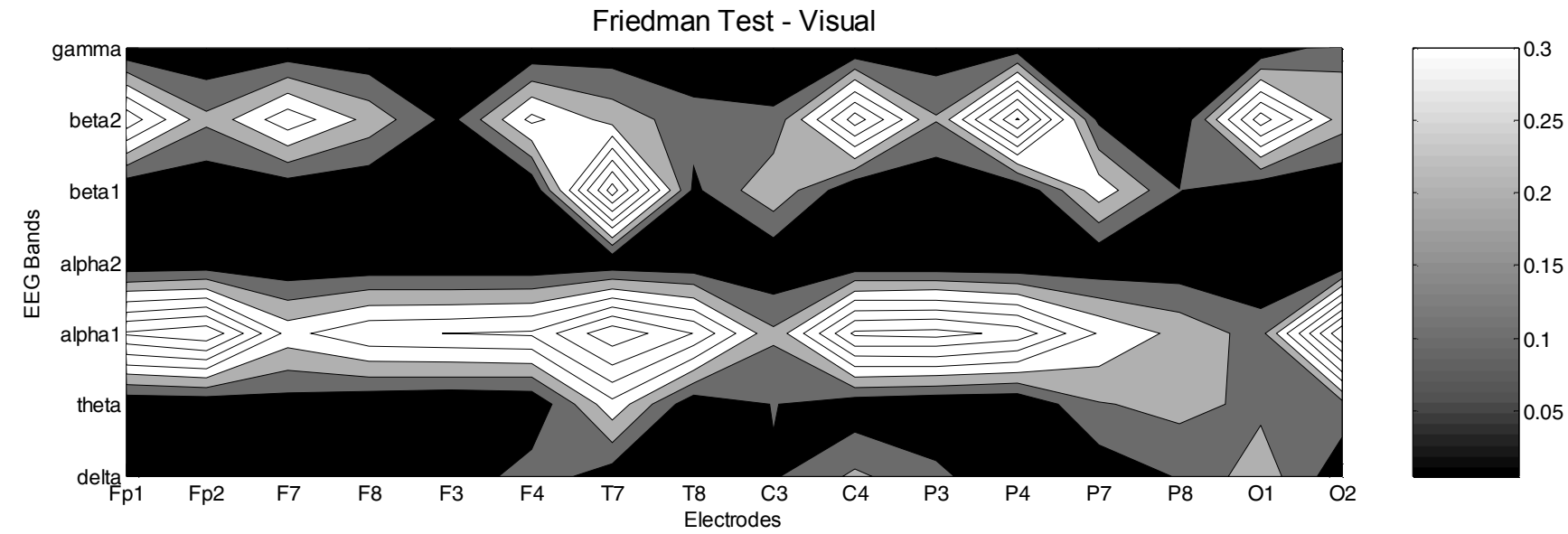

Figure 1. Statistical Spectral Plot of Friedman Rank Test results for Visual Stimulation. The X-axis Represents the Electrode Positions, Y-axis, 7 EEG Bands and Legend Represents the Alpha Rate (p-value).

\section{CONCLUSION}

In comparison to 1 and 3-Way repeated-measures ANOVA tests conducted, only the Theta, Alpha2 and Gamma bands exhibited a significant difference under visual stimuli at extremely low frequencies of 50, 16.66, 13, 10, 8.33 and $4 \mathrm{~Hz}$. There was a clear indication of slight difference in statistical analysis of EEG results between the 1 and 3-way repeated-measures of ANOVA. In order to examine the photic driving or entrainment, further analysis on coherence between stimuli and EEG narrow bands should be performed in the future.

\section{REFERENCES}

[1] S. Ahmed, N.L. Cutter, A.J. Lewy, V.K. Bauer, R.L. Sack, M.S. Cardoza, "Phase response curve of low-intensity green light in winter depressives", Sleep Research, vol. 24, 1995.

[2] P. D. Jaillet, "Apparatus and method for changing critical brain activity using light and sound" U.S. Patent 20020198577, 2002.

[3] H.A. Thomas Jr., Circadian Rhythms and Shift Work, American College of Emergency Physicians, 2001. pp. 1-4.

[4] H. Senger, Blue Light Responses: Phenomena and Occurrence in Plants and Microorganisms. Florida, USA: CRC Press, Boca Raton. 1987, pp. 88-96.

[5] C. Cajochen, D.P. Brunner, K. Krauchi, P. Graw, A.W-Justice, "EEG and Subjective Sleepiness during Extended Wakefulness in Seasonal Affective Disorder: Circadian and Homeostatic Influences", Biol. Psychiatry, vol. 47, pp. 610-617, 2000.

[6] H.R. Wright, L.C. Lack, K.J. Partridge, "Light Emitting Diodes can be used to Phase Delay the Melatonin Rhythm", Journal of Pineal Research, vol. 31(4), p. 350, 2001.

[7] T. Kawaguchi, H. Jijiwa, S. Watanabe, "The Dynamics of Phase Relationships of Alpha Waves during Photic Driving", Electroencephalography and Clinical Neurophysiology, vol. 87, pp. 88-96, 1993.

[8] Y. Wada, Y. Nanbu, R. Kadoshima, Z-Y. Jiang, Y. Koshino, T. Hashimoto, "Interhemispheric EEG coherence during photic stimulation: sex differences in normal young adults", International Journal of Psychophysiology, vol. 22, pp. 45-51, 1996.

[9] D. Cvetkovic, I. Cosic, "The induced rhythmic oscillations of neural activity in the human brain" in 2004 Proc. IASTED (BIOMED) Conf., ACTA Press, (CD-ROM).
[10] D. Cvetkovic, "Electromagnetic and Audio-Visual Stimulation of the Human Brain at Extremely Low Frequencies" Ph.D. dissertation, School of Electrical and Computer Engineering, RMIT Univ., Melbourne, Australia, 2005.

[11] D. Regan, Human Brain electrophysiology: evoked potentials and evoked magnetic fields in science and medicine, New York: Elsevier Publisher, 1989.

[12] R.B. Silberstein, "Steady-state visually evoked potential topography associated with a visual vigilance task", Brain Topography, vol. 3, pp. 337-347, 1990. 